\title{
Proteasome Inhibition Triggers Activity-Dependent Increase in the Size of the Recycling Vesicle Pool in Cultured Hippocampal Neurons
}

\author{
Kristen Willeumier, ${ }^{1}$ Stefan M. Pulst, ${ }^{1,2}$ and Felix E. Schweizer ${ }^{1}$ \\ ${ }^{1}$ Department of Neurobiology, David Geffen School of Medicine at University of California, Los Angeles, Los Angeles, California 90095, and ${ }^{2}$ Division of \\ Neurology, Cedars-Sinai Medial Center, Los Angeles, California 90048
}

\begin{abstract}
The ubiquitin proteasome system, generally known for its function in protein degradation, also appears to play an important role in regulating membrane trafficking. A role for the proteasome in regulating presynaptic release and vesicle trafficking has been proposed for invertebrates, but it remains to be tested in mammalian presynaptic terminals. We used the fluorescent styrylpyridinium dye FM4-64 to visualize changes in the recycling pool of vesicles in hippocampal culture under pharmacological inhibition of the proteasome. We found that a $2 \mathrm{~h}$ inhibition increases the recycling pool of vesicles by $76 \%$, with no change in the rate or total amount of dye release. Interestingly, enhancement did not depend on protein synthesis but did depend on synaptic activity; blocking action potentials during proteasome inhibition abolished the effect whereas increasing neuronal activity accelerated the effect with an increased recycling pool evident after $15 \mathrm{~min}$. We propose that the proteasome acts as a negative-feedback regulator of synaptic transmission, possibly serving a homeostatic role.
\end{abstract}

Key words: synaptic transmission; proteasome; vesicle pools; homeostasis; FM assay; hippocampus

\section{Introduction}

Neurotransmitter release from presynaptic nerve terminals is strongly regulated by both positive as well as negative factors. Calcium clearly plays a crucial role in initiating fusion of synaptic vesicles with the plasma membrane, but a large collection of proteins, lipids and higher order complexes orchestrates the approach of vesicles to the fusion site, readies them for fusion, hampers fusion at inopportune times and accelerates fusion after calcium entry. After fusion, additional protein complexes, possibly sharing crucial components such as synaptotagmin (Poskanzer et al., 2003), then initiate and modulate vesicle endocytosis. Each one of these steps appears to be regulated by a complex set of protein and lipid interactions that are governed at least in part by phosphorylation and also by the presence or absence of particular proteins. Neurons can thus regulate synaptic transmission by modifying the protein composition found at a particular synaptic terminal either by protein synthesis (Beaumont et al., 2001; Giu-

\footnotetext{
Received April 19, 2006; revised Sept. 20, 2006; accepted Sept. 21, 2006.

This work was supported by a National Research Service Award (K.W.), a grant from the American Parkinson Disease Association (F.E.S.), National Institutes of Health Grants NS41317 (F.E.S.) and NS048083 (S.M.P.), and Udall Parkinson's Disease Center Grant NS038367, project 4 (F.E.S., S.M.P.). We are grateful to David Savin for his thorough work on the data analysis. We also thank Drs. Alberto Cruz-Martín, Peyman Golshani, Fred Gregory, Nigel Maidment Tom Otis, and Catia Sternini for critical review of this manuscript, and all members of the Neurobiology Department for feedback and discussions.

Correspondence should be addressed to Dr. Felix E. Schweizer, Department of Neurobiology, David Geffen Schoo of Medicine at University of California, Los Angeles, 650 Charles E. Young Drive South, CHS 63-323, Los Angeles, CA 90095-1763. E-mail: felixs@ucla.edu.

DOI:10.1523/JNEUROSCI.1684-06.2006

Copyright $\odot 2006$ Society for Neuroscience ～0270-6474/06/2611333-09\$15.00/0
}

ditta et al., 2002; Wu et al., 2005) or degradation (Bingol and Schuman, 2005).

The ubiquitin proteasome system (UPS) (Glickman and Ciechanover, 2002) is emerging as a powerful modulator of synaptic transmission (Murphey and Godenschwege, 2002; Cline, 2003; Hegde, 2004; Yi and Ehlers, 2005; Patrick, 2006). For example, UPS dependent degradation of important postsynaptic components has been reported (Burbea et al., 2002; Colledge et al., 2003; Ehlers, 2003; Patrick et al., 2003; Juo and Kaplan, 2004). Furthermore, the proteasome itself seems to be trafficked in and out of postsynaptic spines in an activity-dependent manner (Bingol and Schuman, 2006).

The proteasome modulates synaptic transmission presynaptically at both the Drosophila neuromuscular junction (NMJ) (Aravamudan and Broadie, 2003; Speese et al., 2003) and the Aplysia sensory-motor synapse (Zhao et al., 2003). This regulation appears to depend on protein kinase A (PKA) activity resulting in an acute enhancement of evoked transmission (Aravamudan and Broadie, 2003; Speese et al., 2003), whereas the increased fiber sprouting and long-term facilitation observed in Aplysia requires additional protein synthesis (Zhao et al., 2003). The proteasome also regulates the level of presynaptic proteins including the vesicle priming protein Dunc-13 (Aravamudan and Broadie, 2003; Speese et al., 2003), the vesicle membrane protein synaptophysin (Wheeler et al., 2002), and the plasma membrane soluble $\mathrm{N}$-ethylmaleimide-sensitive factor attachment protein (SNAP) receptors (SNAREs) syntaxin (Chin et al., 2002) and SNAP-25 (Ma et al., 2005).

Our studies are focused on elucidating the role of the protea- 
some in the vesicle recycling pathway. Using the styryl dye FM4-64 in dissociated culture, we visualized changes in the recycling pool of vesicles in the presence or absence of the proteasome inhibitors clasto-lactacystin $\beta$-lactone or epoxomicin. We demonstrate that inhibition of the proteasome increases the recycling pool in an activity-dependent manner and that this regulation can be modulated by second messenger pathways. Our data show that the proteasome can dynamically regulate synaptic transmission and suggest that the proteasome serves a regulatory feedback role in the presynaptic terminal.

\section{Materials and Methods}

Cell culture. Hippocampal cultures from the CA1-CA3 regions of newborn rat pups (postnatal day 0-3) were prepared as described previously (Sippy et al., 2003). The CA1-CA3 region of the hippocampus was dissected from Sprague Dawley rats in HBSS (Life Technologies, Gaithersburg, MD) containing 20\% fetal bovine serum (FBS; Hyclone, Logan, UT). The tissue was digested for $5 \mathrm{~min}$ with $10 \mathrm{mg} / \mathrm{ml}$ trypsin, and mechanically dissociated with a siliconized Pasteur pipette in $0.5 \mathrm{mg} / \mathrm{ml}$ DNase. After centrifugation, the cells were plated at a density of 30,000$50,000 / \mathrm{cm}^{2}$ on glass coverslips inside an 8 - $\mathrm{mm}$-diameter cloning cylinder on glass coverslips coated with Matrigel (Life Technologies). Cells were grown in $50 \mu \mathrm{l}$ Minimal Essential Medium (MEM; Life Technologies) supplemented with $0.5 \%$ glucose, $100 \mathrm{mg} / \mathrm{L}$ bovine transferrin (Calbiochem, La Jolla, CA), 24 mg/L insulin, 2 mm Glutamax-1 (Invitrogen, Carlsbad, CA) and 10\% FBS (Hyclone). 24-48 h after plating $50 \mu \mathrm{l}$ of with MEM supplemented as above but containing no FBS, $4 \%$ B-27 (Invitrogen) and $8 \mu \mathrm{M}$ cytosine $\beta$-D-arabinofuranoside (ARA-C; Sigma, St. Louis, MO) for a final concentration of 5\% FBS, 2\% B27 and $4 \mu \mathrm{M}$ ARA-C. Cultures were maintained at $37^{\circ} \mathrm{C}$ in a $95 \%$ air $/ 5 \% \mathrm{CO}_{2}$ humidified incubator for 12-21 d before use.

FM 4-64 assay to measure vesicle recycling; imaging, and analysis. Coverslips with cultured neurons [12-21 d in vitro (DIV)] were mounted on a fast-flow perfusion chamber on the stage of a laser-scanning confocal microscope [Zeiss (Oberkochen, Germany) LSM 410 attached to a Zeiss Axiovert 100 , using a $100 \times, 1.3$ numerical aperture oil-immersion objective]. The total chamber volume of $100 \mu \mathrm{l}$ was perfused at a rate of 5 $\mathrm{ml} / \mathrm{min}$ allowing for fast switching of external solutions. Control external solution contained the following (in mM): $119 \mathrm{NaCl}, 5 \mathrm{KCl}, 25 \mathrm{HEPES}, 30$ D-glucose, $2 \mathrm{CaCl}_{2}, 2 \mathrm{MgCl}_{2}$, pH 7.2 with $\mathrm{NaOH}, 310$ mOsm. High K ${ }^{+}$ external solution contained the following (in $\mathrm{mm}$ ): $34 \mathrm{NaCl}, 90 \mathrm{KCl}, 25$ HEPES, 30 D-glucose, $2 \mathrm{CaCl}_{2}, 2 \mathrm{MgCl}_{2}$, pH 7.2 with $\mathrm{NaOH}, 310 \mathrm{mOsm}$. Neurons were challenged for 1 min with high- $\mathrm{K}^{+}$solution in the presence of $15 \mu \mathrm{M}$ FM4-64 or SynaptoRed. Neurons were then washed for 10 min in control external solution to remove any nonendocytosed dye. Advasep $(100 \mu \mathrm{M})$ was perfused for $30 \mathrm{~s}$ to quench additional dye not taken up by endocytosis. After washing, the cells were challenged a second time for $1 \mathrm{~min}$ with high- $\mathrm{K}^{+}$solution without fluorescent dye (destaining). Confocal images of $256 \times 256$ pixels with a $3 \times$ zoom $(0.17$ $\mu \mathrm{m} /$ pixel) were acquired every $2 \mathrm{~s}$ during the last minute of the wash and then during the second high- $\mathrm{K}^{+}$challenge (identical gain and contrast settings were used throughout the study).

The area for imaging was chosen to contain a meshwork of well defined dendrites and no clusters of cell bodies. Acquired images were analyzed offline using ImageJ software (http://rsb.info.nih.gov/ij/) and the built-in particle analyzer function was used to track the fluorescence intensity of all pixel clusters within an image that contained 6-50 pixels with an average intensity above a user-set threshold. These criteria marked $\sim 40$ pixel clusters per experiment, largely independent of the experimental condition (see Fig. 2) [control (Ctrl), $37.3 \pm 1.7$; clastolactacystin $\beta$-lactone treated (Clast), $45.2 \pm 1.9$; DMSO, $39.9 \pm 3.2$; epoxomicin (Epox), $42.3 \pm 3.2$ ]. The small increase in the number of clusters in response to proteasome inhibition, although not seen in all experiments, might indicate an additional effect on synapse number that was not further explored, but would not alter any of our conclusions. To analyze these clusters, a mask was created by subtracting the average of the last 30 images from an average of the first 30 images in a series. Particle tracker then identified the clusters in the subtracted image. All pixels belonging to a cluster and a ring of pixels around each cluster were then set to the numerical value 1 whereas all other pixels were assigned the value 0 . This mask was then multiplied with each original image in a series, effectively setting the background to 0 without changing the clusters and allowing for some lateral movement of the clusters between images. This simple procedure allows for a relatively unbiased and efficient analysis of the experiments. The analysis was performed blind to experimental condition. The initial fluorescence intensity, the time course of fluorescence decay and the fraction of the initial fluorescence that could be released were determined (see Fig. 1). Rare experiments showed no dye uptake or no dye release and were excluded from additional analysis.

Values from the $\sim 40$ pixel clusters analyzed in a single experiment $(n)$ were averaged and an exemplar is shown in Figure 1. The average of these averages from repeated experiments is reported as the mean \pm SEM, with $n$ representing the number of independent experiments. All experiments were repeated with at least three independent batches of cell cultures. Data were compared using the Student's $t$ test and resampling statistics (Efron and Tibshirani, 1991). For multiple comparisons the data were analyzed by two-way ANOVA followed by Tukey's post hoc analysis to determine individual group differences.

Proteasome inhibitor treatment. Cultures were visually inspected to ensure healthy appearance before the experiment. The proteasome inhibitors clasto-lactacystin $\beta$-lactone, epoxomicin, or MG-132 (Boston Biochem, Cambridge, MA), were solubilized in 10\% DMSO (Sigma) and added to the cultures to give a final concentration of $10 \mu \mathrm{M}$ for clastolactacystin $\beta$-lactone, epoxomicin and $50 \mu \mathrm{M}$ for MG-132 in 1\% DMSO unless otherwise indicated. Control cultures were exposed to $1 \%$ (DMSO) for identical durations. The reversible inhibitor MG-132 was also added to all solutions during the FM4-64 experiments. Control experiments showed that DMSO at concentrations of $0.1,1$ and $2 \%$ was without effect on the measured signals as compared with nontreated controls (Table 1).

Chemicals. The chemicals used in these experiments and their sources are as follows: FM4-64 (Invitrogen), Advasep (CyDex, Lenexa, KS), H-89, emetine (Calbiochem) Sp-cAMPS, Rp-cAMPS, cycloheximide (Biomol, Plymouth Meeting, PA), and forskolin (Sigma). In some experiments, the structurally identical Synaptored-C2 (Biotium, Hayward, CA) was used instead of FM4-64.

\section{Results}

Acute inhibition of the proteasome increases the recycling vesicle pool in primary neuronal cultures

We studied synaptic transmission in primary cultures of hippocampal neurons obtained from 0 - to 2-d-old rat pups. After $2-3$ weeks in culture, the neurons were treated with proteasome inhibitors and the fluorescent membrane marker FM4-64 was used to quantify the presynaptic components of synaptic transmission (Betz and Bewick, 1992; Ryan et al., 1993). To stimulate exocytosis and subsequent dye uptake, cells were depolarized with a hyperkalemic solution $(90 \mathrm{~mm} \mathrm{KCl})$ for $1 \mathrm{~min}$ in the presence of $15 \mu \mathrm{M}$ FM4-64, a stimulus paradigm thought to recruit 
A

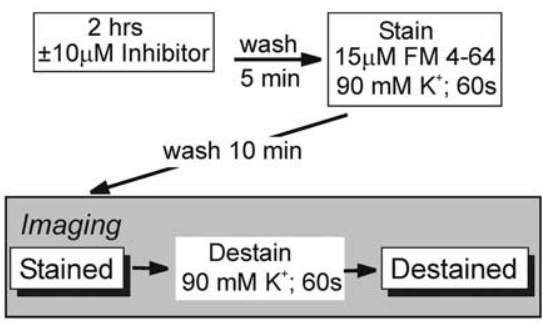

B

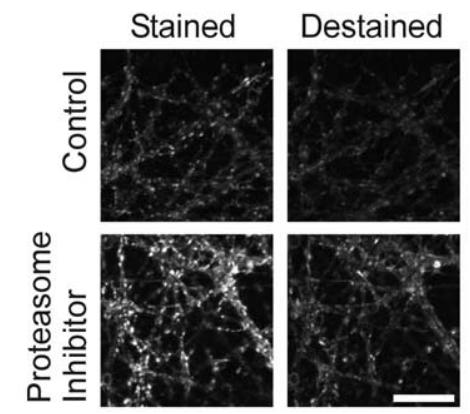

C

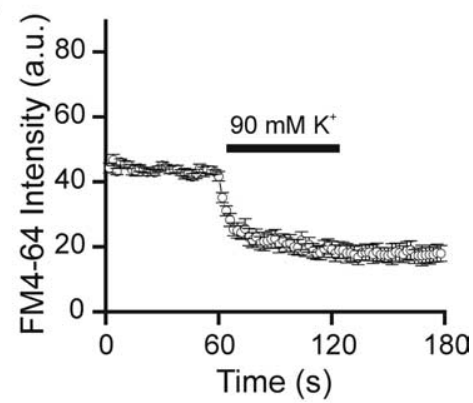

D

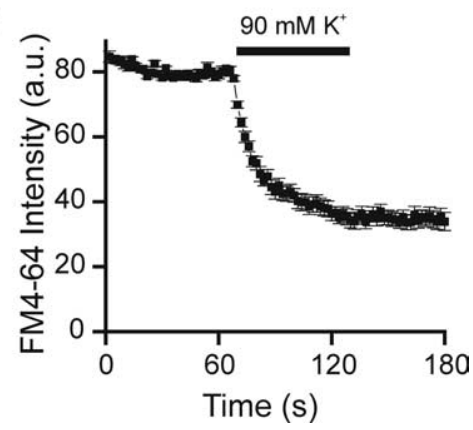

Figure 1. Clasto-lactacystin $\beta$-lactone increases the FM4 - 64 dye uptake. $A$, Schematic of FM4-64 experiments to measure presynaptic function (for details, see Materials and Methods and Results). $\boldsymbol{B}$, Left, Fluorescence images of cultured hippocampal neurons taken at the end of the 10 min wash period (stained) indicate that control neuronal terminals (top; Control) show less dye uptake than clasto-lactacystin $\beta$-lactone-treated neurons (bottom; Proteasome Inhibitor). Right, The same respective fields after destaining using a 60 s hyperkalemic treatment in the absence of FM4-64. Scale bar, $15 \mu \mathrm{m}$. C, D, Plot of FM4- 64 fluorescence intensity over time. The fluorescence intensities of the $\sim 40$ puncta in $\boldsymbol{B}$ were analyzed in all images taken and the average \pm SEM is plotted against time for control ( $\boldsymbol{C}$ and clasto-lactacystin $\beta$-lactone-treated cultures ( $\boldsymbol{D}$; for details, see Materials and Methods). From these graphs, FM4-64 uptake, the kinetics of dye release, and the percentage of dye released were calculated (Ctrl, $39.1 \pm$ 3.7 a.u., 15.9 s, $61 \%$; Clast, $79.6 \pm 0.2$ a.u., 19.6s, 57\%). Note the close similarities between these two exemplar datasets, except for the amount of initial dye uptake. Each graph in this figure depicts a single experiment $n$ (see Materials and Methods).

the entire pool of actively recycling vesicles (Ryan et al., 1993). After a 10 min washing step, fluorescent spots were prominently visible (Fig. $1 B$, stained) indicating sites of active membrane turnover. These sites represent presynaptic terminals based on immunohistochemistry (Ryan et al., 1993) and EM examination (Harata et al., 2001; Schikorski and Stevens, 2001). Images were taken every $2 \mathrm{~s}$ and, after a $1 \mathrm{~min}$ baseline period, the neurons were depolarized with a second 1 min application of a hyperkalemic solution containing no dye. This stimulates the fusion of vesicles that have previously taken up the dye, leading to a loss of fluorescence from active synapses (Fig. $1 B$, destained). Any endocytic event occurring during the $1 \mathrm{~min}$ dye application will trap dye; however, the optical signals are dominated by the large scale and fast, stimulus-synchronized time course of vesicle release and uptake at the presynaptic terminal. The recorded signals thus reflect almost exclusively presynaptic events. We used ImageJ to quantify the FM4-64 fluorescence intensity of individual spots over time (see Materials and Methods). Typically, $\sim 40$ individual spots were analyzed per trial and the average intensity and SEs were plotted against time as shown for an example in Figure 1C. The total dye uptake, the time course of release and the amount of dye released was then determined. These numbers were regarded as representing a single experiment $n(n=$ number of individual coverslips). In each series of experiments, at least one control culture was compared with at least one treated culture (protease inhibitors, kinase inhibitors, etc.). Experimental data were obtained from at least three independent cultures. On average, $50.0 \pm 1.0 \%$ of the loaded FM dye was released during the second high potassium challenge (Fig. $2 C)(n=$ 56) with a time constant of $23.6 \pm 1.0 \mathrm{~s}$ $(n=39)$ (Fig. 2E).

After a $2 \mathrm{~h}$ treatment with the proteasome inhibitor clasto-lactacystin $\beta$-lactone $(10 \mu \mathrm{M})$ the amount of dye loading increased dramatically relative to untreated control cultures (Fig. $1 B-D$ ). In our entire series of experiments, we observed a $76 \%$ increase in dye uptake after proteasome inhibitor treatment (Fig. $2 A, B)\left[{ }^{\star} p<\right.$ 0.0001; Ctrl, $59.5 \pm 2.3$ a.u., $n=56$ (2089 boutons); Clast, $104.9 \pm 3.7$ a.u., $n=48$ (2169 boutons)]. Both the kinetics and relative amount of dye released were not affected by proteasome inhibition (Fig. $2 \mathrm{C}-$ $F)$. These results were robust for all paired experimental sets (Fig. 1) as well as for the entire data set (Fig. 2). At lower concentrations $(1 \mu \mathrm{M})$ (data not shown), clastolactacystin $\beta$-lactone was less effective and gave more variable results, whereas higher concentrations $(100 \mu \mathrm{M})$ appeared somewhat toxic (as determined by propidium iodine staining) (data not shown). The widely used concentration of $10 \mu \mathrm{M}$ clastolactacystin $\beta$-lactone (Zhao et al., 2003) was therefore chosen as a standard concentration.

Although clasto-lactacystin $\beta$-lactone is a highly specific, nonreversible proteasome inhibitor (Fenteany et al., 1994), it was important to confirm the results with another specific but structurally unrelated proteasome inhibitor. As shown in Figure 2 , a $2 \mathrm{~h}$ treatment with $10 \mu \mathrm{M}$ epoxomicin yielded results virtually identical to those obtained with clasto-lactacystin $\beta$-lactone, although the effect of epoxomicin was slightly smaller $(p<0.03)$. We observed a $67 \%$ increase in dye uptake $\left[{ }^{*} p<0.0001\right.$; Ctrl, $53.5 \pm 3.8$ a.u., $n=19$ (758 boutons); Epox, $89.6 \pm 6.11$ a.u., $n=$ 19 (804 boutons)] with no change in the kinetics or relative amount of dye released (Fig. $2 C-F$ ). There also was no change in the average number of FM spots per image in response to the $2 \mathrm{~h}$ exposure to proteasome inhibitors (data not shown). We further corroborated our results by using a more common, but possibly less-specific proteasome inhibitor, MG-132, at $50 \mu \mathrm{m}$. Because this inhibitor is reversible, we included it in all staining and destaining solutions in addition to the $2 \mathrm{~h}$ preincubation. As with the other two inhibitors, we observed an increase in dye uptake $(p<0.02$; Ctrl, $43.5 \pm 2.5$ a.u., MG-132, $57.2 \pm 4.9 n=9)$. Because acute proteasome inhibition with three unrelated inhibitors increased the amount of dye uptake with no change in the kinetics or the relative amount of dye release, we conclude that proteasome inhibition increases the number of synaptic vesicles in the pool of recycling vesicles without directly affecting the exocytotic process.

We next investigated the time course of the observed increase in the recycling pool of synaptic vesicles. Whereas a $15 \mathrm{~min}$ treatment was not enough to reveal a statistically significant effect, 60 min of proteasome inhibitor exposure was sufficient to trigger a maximal increase which was sustained for at least $5 \mathrm{~h}$ (Fig. $3 A$ ). A $2 \mathrm{~h}$ wash between treatment with the irreversible inhibitors and the FM assay did not diminish the effect. However, preliminary 
A

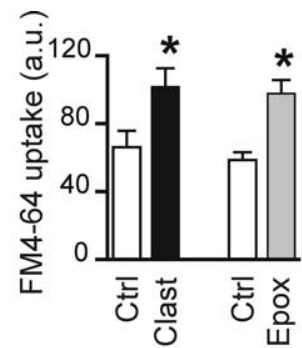

C

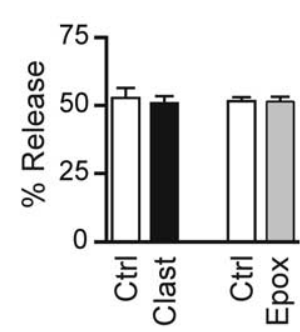

E

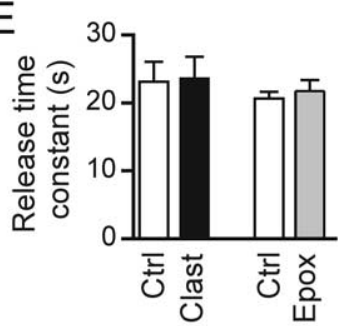

$\mathrm{B}$
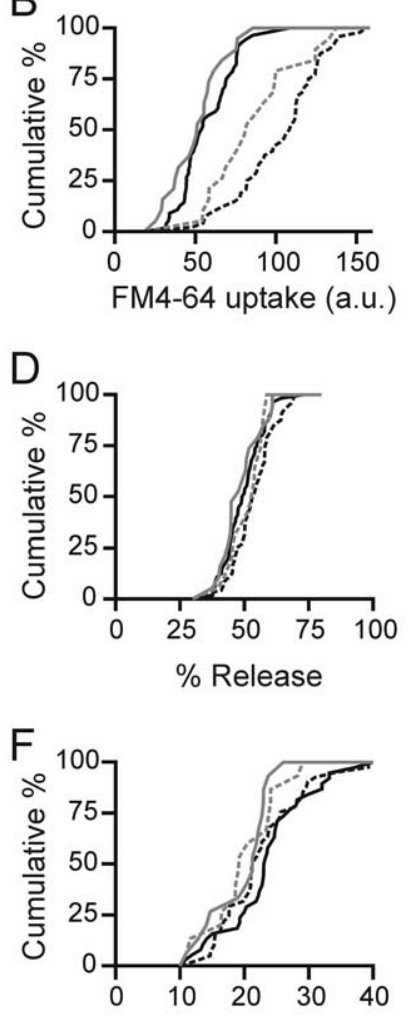

Release time constant (s)
Figure 2. Proteasome inhibitors significantly increase FM4-64 uptake without altering the kinetics or the relative amount of release. $A$, Dye uptake was quantified as in Figure 1 and is shown as mean \pm SEM. Open bars, Control; black bars, clasto-lactacystin $\beta$-lactone; gray bars, epoxomicin. ${ }^{*} p<0.001$, Student's $t$ test. $\boldsymbol{B}$, Same data as in $\boldsymbol{A}$ but plotted as a cumulative distribution. Solid lines, Controls; black dotted line, clasto-lactacystin $\beta$-lactone; gray dotted line, epoxomicin. Although the means are not significantly different $(\boldsymbol{A})$, the cumulative distribution indicates that the median increase in clasto-lactacystin $\beta$-lactone is larger than in control. ${ }^{*} p<0.01$, resampling statistics. $C, D$, Amount of dye that was released in percentage of dye uptake (same setup as in $\boldsymbol{A}, \boldsymbol{B}$ ). $\boldsymbol{E}, \boldsymbol{F}$, Kinetics of dye release (same setup as in $\boldsymbol{A}, \boldsymbol{B}$ ).

experiments with the reversible MG-132 indicate that the number of recycling vesicles returns to control levels in $<2 \mathrm{~h}$ (data not shown). For the present study, we focused on the acute effects of irreversible proteasome inhibitors and chose treatments of 15 min and $2 \mathrm{~h}$ as our standard. All experiments were performed with neuronal cultures grown for 12-21 DIV after synapse formation and substantial maturation has occurred (Renger et al., 2001). Plotting dye uptake against DIV for control cultures revealed no significant increase over the time period tested (Fig. 3B, open circles). However, in clasto-lactacystin $\beta$-lactone-treated cultures, dye uptake increased substantially over the same period. Thus, although basic synaptic properties as tested by a hyperkalemic challenge are "mature" at 12 DIV, further development is required for the proteasome to act as a modulator of the recycling vesicle pool.

Inhibition of the proteasome blocks protein degradation and, thus, increases the level of some proteins. Such an increase in protein levels might additionally depend on synthesis of new proteins. We tested this hypothesis by treating neuronal cultures with $100 \mu \mathrm{M}$ of either cycloheximide or emetine during proteasome inhibition. Neither drug affected dye uptake (and thus vesicle recycling) on their own nor in combination with the proteasome inhibitor clasto-lactacystin $\beta$-lactone, which still increases the recycling pool (Fig. $3 C, D)\left(F_{(1,32)}=1.3 ; p>0.2\right)$. This result shows that the effect of proteasome inhibition is independent from the synthesis of novel proteins. It has been shown previously that proteasome inhibition over a $24 \mathrm{~h}$ period causes a protein synthesis dependent increase in synaptic transmission in Aplysia (Zhao et al., 2003). Our results are consistent with findings in Drosophila where proteasome inhibition for $1 \mathrm{~h}$ increased the release probability independent of new protein synthesis (Speese et al., 2003). Together these results show that protein levels at the presynaptic terminal can be altered by proteasomal function alone, whereas longer term changes that include structural rearrangements (Zhao et al., 2003) depend on additional protein synthesis.

\section{Proteasome inhibition-mediated increase in the recycling vesicle pool depends on neuronal activity}

Although the regulation of proteasomal activity is not well understood, previous experiments suggest that it might be modulated by neuronal activity; acute depolarization of synaptosomes triggers a rapid downregulation of protein ubiquitination (Chen et al., 2003), whereas changes in neuronal activity over hours to days alters the levels of ubiquitinated proteins in the postsynaptic density (Ehlers, 2003). Because hippocampal cultures show high levels of intrinsic activity, it seemed possible that the time course of the effect of proteasome inhibition (Fig. $3 A$ ) might be affected by neuronal activity. To test this, neuronal activity was increased during a short, 15 min proteasome inhibitor treatment (Fig. $4 \mathrm{~A}$, high activity) or decreased during the standard $2 \mathrm{~h}$ long treatment (Fig. $4 A$, low activity). The effect on the pool size was then determined using the standard solutions and protocols (Figs. 1 $A, 4 A$ ). To increase neuronal activity, inhibition was blocked by $100 \mu \mathrm{M}$ picrotoxin and synaptic activity was enhanced by elevating the extracellular $\mathrm{Ca}^{2+}$ concentration from $2 \mathrm{~mm}$ to $5 \mathrm{~mm}$. To lower neuronal activity, action potentials were blocked by $500 \mathrm{nM}$ tetrodotoxin (TTX) in the presence of selective antagonists for AMPA and NMDA receptors [6-cyano-7-nitroquinoxaline-2,3dione (CNQX; $10 \mu \mathrm{M}$ ) and 2-amino-5-phosphonopentanoic acid (APV; $50 \mu \mathrm{M})$, respectively]. Dye uptake and release was then assessed using the identical, standard external solutions for all conditions. Elevation or blockade of neuronal activity in the absence of proteasome inhibitor (Fig. $4 C, D$ ) was without effect on the size of the recycling pool. However, whereas a 15 min exposure to proteasome inhibitors failed to elicit an effect (Fig. 3A, $4 C, D)$, high activity for $15 \mathrm{~min}$ in the presence of either clastolactacystin $\beta$-lactone (Fig. 4C) or epoxomicin (Fig. 4D) increased the size of the recycling pool to levels comparable with those achieved with a $2 \mathrm{~h}$ exposure to proteasome inhibitor alone (5Ca ${ }^{+2} /$ Clast, $94.6 \pm 7.4$ a.u., $n=24 ; 2$ h Clast, $96.6 \pm 6.5$ a.u., $n=30$; and $5 \mathrm{Ca}^{+2} /$ Epox, $78.8 \pm 14.9$ a.u., $n=9 ; 2 \mathrm{~h} \mathrm{Epox,} 87.1 \pm$ 8.0 a.u., $n=16$ ) (Fig. $4 C, D$ ). Consistent with activity dependence, blocking neuronal activity during a $2 \mathrm{~h}$ exposure to proteasome inhibitors completely blocked the increase in the vesicle pool size (Fig. 4C,D). Together, these results reveal an activitydependent effect of proteasome inhibition and suggest that the proteasome might act as a regulatory feedback control mechanism to contain exocytotic activity (see Discussion).

\section{Potential overlap between cAMP/PKA-mediated and proteasome-dependent increase in the recycling pool of vesicles}

The recycling pool of vesicles appears to be refilled from the reserve pool, possibly through a cAMP/PKA-dependent pathway, as suggested by the Drosophila mutants dunce and rutabaga that have deficiencies in the cAMP pathway (Kidokoro et al., 


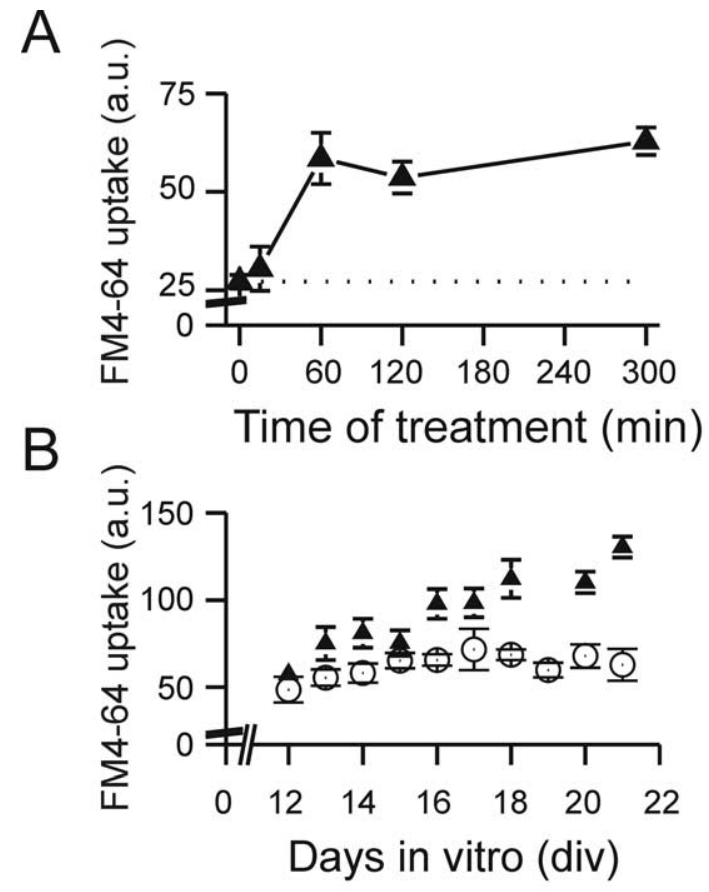

C
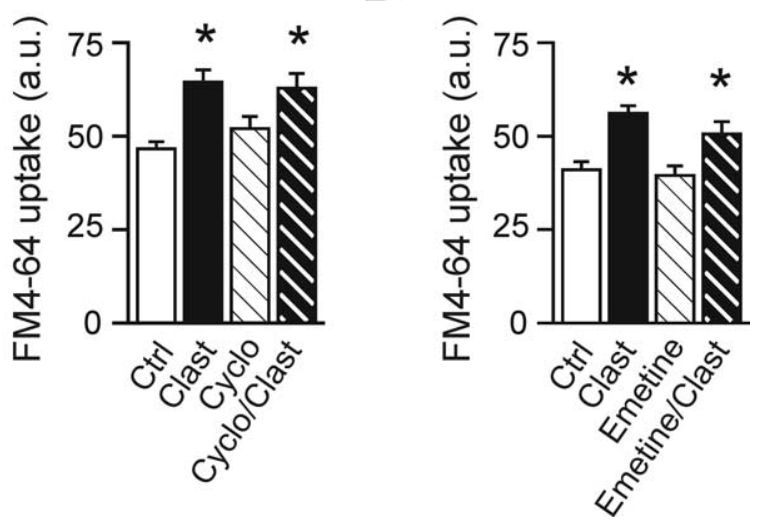

Figure 3. Effect of proteasome inhibition is enhanced in older cultures, is stable over time, and does not depend on protein synthesis. $\boldsymbol{A}$, Clasto-lactacystin $\beta$-lactone was applied for various lengths of time and the average dye uptake is plotted over time as mean $\pm \mathrm{SEM}$ ( 0 min, $41.7 \pm 1.4$ a.u., $n=21 ; 15 \min , 44.4 \pm 4.5$ a.u., $n=13 ; 60 \mathrm{~min}, 66.8 \pm 5.2$ a.u., $n=13 ; 120$ $\min , 62.9 \pm 3.2$ a.u., $n=25 ; 300 \min , 70.31 \pm 2.8$ a.u., $n=4)$. $\boldsymbol{B}$, FM4 -64 dye uptake as a function of days in culture and plotted as mean \pm SEM (day $12 \mathrm{Ctrl}, 48.6 \pm 7.3$ a.u., $n=3$; Clast, 57.4 a.u., $n=1$; day 13 Ctrl, $55.5 \pm 4.6$ a.u., $n=18$; Clast, $75.0 \pm 9.5$ a.u., $n=9$; day 14 Ctrl, $58.1 \pm 5.5$ a.u., $n=8$; Clast, $81.0 \pm 8.3$ a.u., $n=6$; day 15 Ctrl, $65.2 \pm 4.4$ a.u., $n=7$; Clast, $75.4 \pm 7.4$ a.u., $n=7$; day 16 Ctrl, $65.6 \pm 3.3$ a.u., $n=12$; Clast, $97.8 \pm 8.6$ a.u., $n=12 ;$ day $17 \mathrm{Ctrl}, 71.7 \pm 11.9$ a.u., $n=7$; Clast, $98.4 \pm 8.3$ a.u., $n=9 ;$ day $18 \mathrm{Ctrl}, 68.6 \pm$ 3.1 a.u., $n=13$; Clast, $112.1 \pm 10.9$ a.u., $n=9$; day 19 Ctrl, $59.8 \pm 4.2$ a.u., $n=15$; Clast, $n=$ 0 , day 20 Ctrl, $67.9 \pm 6.7$ a.u., $n=8$; Clast, $110.1 \pm 6.2$ a.u., $n=20$; day 21 Ctrl, $62.8 \pm$ 9.1 a.u., $n=6$; Clast, $130.4 \pm 6.0$ a.u., $n=9$ ). Controls (open circles) and $2 \mathrm{~h}$ proteasome inhibitor-treated cultures (filled triangles) were analyzed. $\boldsymbol{C}, \boldsymbol{D}$, The $2 \mathrm{~h}$ increase in the vesicle recycling pool is not dependent on protein synthesis. C, Cultures were treated with vehicle ( $1 \%$ DMSO) or the protein synthesis inhibitor cycloheximide (Cyclo) $(100 \mu \mathrm{m})$ for $2 \mathrm{~h}$ in the absence or presence of clasto-lactacystin $\beta$-lactone $(10 \mu \mathrm{M})$. Average dye uptake is plotted as mean \pm SEM (Ctrl, $46.56 \pm 1.97 ;$ Clast, 64.45 \pm 3.26; Cyclo, $52.02 \pm 3.22 ;$ Cyclo/Clast, $62.81 \pm 3.93$, $n=9$ in all conditions). Two-way ANOVA followed by Tukey's post hoc analysis at $p<0.05$ revealed a significant difference in clasto-lactacystin $\beta$-lactone treated cultures (*relative to $C$ trl) with no effect of cycloheximide. $\boldsymbol{D}$, Cultures were also treated with the irreversible protein synthesis inhibitor emetine $(100 \mu \mathrm{m})$ for $2 \mathrm{~h}$ in the absence or presence of clasto-lactacystin $\beta$-lactone $(10 \mu \mathrm{m})$. Average dye uptake is plotted as mean \pm SEM (Ctrl, $41.03 \pm 2.13$; Clast, $56.12 \pm 2.02$; Emetine, $39.5 \pm 2.56$; Emetine/Clast, $50.54 \pm 3.35, n=9$ in all conditions). Two-way ANOVA followed by Tukey's post hoc analysis at $p<0.05$ again revealed a significant difference in clasto-lactacystin $\beta$-lactone treated cultures (*relative to $(\mathrm{tr} r)$ ) with no effect of emetine.
2004). Furthermore, the cAMP/PKA-dependent pathway can regulate the activity of the UPS directly (Kotani et al., 1998; Upadhya et al., 2006) and phosphorylation by PKA can alter the proteasomal degradation rate of the phosphorylated protein (Gastel et al., 1998; Schomerus et al., 2000). We thus addressed the hypothesis that the CAMP-PKA pathway intersects with the proteasome-dependent recruitment of vesicles by either blocking or stimulating the cAMP-PKA pathway. To block PKA, neuronal cultures were preincubated for one $h$ with the PKA inhibitors H-89 $(20 \mu \mathrm{M})$ or Rp-cAMPS $(100 \mu \mathrm{M})$ and then treated with inhibitors and clasto-lactacystin $\beta$-lactone or inhibitors alone for another $2 \mathrm{~h}$. Whereas $\mathrm{H}-89$ slightly increased the recycling pool of vesicles relative to control (Ctrl, $55.4 \pm 4.6$ a.u., $n=7$; H-89, $74.03 \pm 6.21$ a.u., $n=9$ ), the competitive PKA inhibitor RpcAMPS had no effect on dye uptake (Ctrl, $51.5 \pm 5.3$ a.u., $n=9$; Rp-cAMPS, $50.2 \pm 2.4$ a.u., $n=9$ ). Importantly, both PKA antagonists completely blocked the effect of proteasome inhibition (Clast, $101.8 \pm 9.2$ a.u., $n=7$; H-89/Clast, $70.6 \pm 7.7$ a.u., $n=$ 10) (Fig. $5 B$ ) (Clast, $76.3 \pm 2.7$ a.u., $n=9$; Rp/Clast, $55.8 \pm$ 4.4 a.u., $n=9$ ) (Fig. 5C). As in the other experiments, neither the absolute amount of dye release nor the rate of dye release was affected by PKA inhibition (data not shown).

To stimulate the cAMP-PKA pathway, we used the adenylyl cyclase activator forskolin, thereby increasing intracellular cAMP concentrations. Alternatively, we used the nonhydrolyzable cAMP-analog Sp-cAMPS, a potent PKA agonist. As with the PKA inhibitors, cultures were pretreated with forskolin $(100 \mu \mathrm{M})$ or Sp-cAMPS $(50 \mu \mathrm{M})$ for $1 \mathrm{~h}$, followed by a $2 \mathrm{~h}$ incubation with forskolin/Sp-cAMPS alone or forskolin/Sp-cAMPS and clastolactacystin $\beta$-lactone. In agreement with previous studies $(\mathrm{Ku}-$ romi and Kidokoro, 1999, 2003), forskolin alone increased the recycling pool of vesicles $\left[{ }^{\star} p<0.05\right.$; Ctrl, $67.4 \pm 2.7$ a.u., $n=23$; forskolin (Forsk), $90.5 \pm 5.6$ a.u., $n=23$ ] (Fig. 5E). This increase was as large as the increase induced by proteasome inhibition alone, yet the combined treatment did not elicit a further enhancement (Clast, $90.6 \pm 7.1$ a.u., $n=18$; Forsk/Clast, $89.2 \pm$ 6.3) (Fig. 5E). Similar results were obtained with the PKA agonist Sp-cAMPS (Ctrl, $50.3 \pm 4.8$ a.u.; Clast, $81.7 \pm 4.1$ a.u.; SpcAMPS, $81.1 \pm 2.8$ a.u.; Sp/Clast, $85.5 \pm 8.8$ a.u.; $n=9$ ) (Fig. $5 F$ ). Again, no change in the rate or amount of dye release was observed (data not shown). This suggests that activation of the cAMP-PKA pathway is capable of recruiting vesicles to the releasable pool, thus increasing the recycling vesicle pool size. In the presence of strong proteasome inhibition, the PKA pathway does not further modulate the vesicle recycling pool. These results suggest that proteasome inhibition and PKA activity cooperate or share common or intersecting pathways in causing an increase in the vesicle recycling pool.

\section{Discussion}

In the present study, we have demonstrated that acute inhibition of the ubiquitin-proteasome system in neurons triggers an increase in the size of the recycling pool of vesicles. Optical measurements obtained by application of hyperkalemic solution showed a $76 \%$ increase in the size of the recycling pool after proteasome inhibitor treatment. Under control conditions, the effect was not apparent after $15 \mathrm{~min}$, but rather developed over 1-2 h of proteasome inhibition and persisted for at least $5 \mathrm{~h}$. The proteasome inhibitor-mediated increase in vesicle pool size was crucially dependent on neuronal activity: increasing neuronal activity during 15 min proteasome inhibitor incubation revealed a robust increase in pool size, whereas blockade of activity during a $2 \mathrm{~h}$ incubation completely abolished the proteasome-inhibition 
effect. Our results demonstrate a potent, activity-dependent regulatory role for the proteasome at synapses. We propose that the proteasome can serve as a presynaptic homeostatic regulator, keeping neurotransmitter release in check during times of heightened activity.

Previous studies have revealed an activity-dependent regulation of synaptic function by the postsynaptic proteasome in hippocampal neurons. Manipulation of network activity for $3 \mathrm{~d}$ alters the ubiquitination and abundance of postsynaptic proteins (Ehlers, 2003). The postsynaptic ubiquitin proteasome system is crucial for receptor endocytosis within minutes of agonist binding (Patrick et al., 2003) and the mobility and localization of the postsynaptic proteasome is controlled by neuronal activity (Bingol and Schuman, 2006). Studies of invertebrate preparations have led to the notion that proteasome inhibition mediates changes of synaptic strength by both presynaptic and postsynaptic mechanisms. In Aplysia, proteasome inhibition for $24 \mathrm{~h}$ in either the presynaptic or the postsynaptic neuron enhances synaptic strength and the enhancement depends on protein synthesis (Zhao et al., 2003). In Drosophila, proteasome inhibition results in increased Dunc-13 levels within $1 \mathrm{~h}$ that do not depend on protein synthesis (Aravamudan and Broadie, 2003), and genetic methods have been used to demonstrate the presynaptic locus of the increased release probability (Speese et al., 2003). In our studies, we used a fluorescent dye uptake assay that monitors almost exclusively presynaptic vesicle recycling and, thus, although the inhibitor was bath applied, the reported effect is presynaptic in nature. However, we cannot presently exclude the possibility that a postsynaptic effect of proteasome inhibition could lead to changes in retrograde signaling that then affect presynaptic function. We also find no evidence for a protein synthesis requirement in increasing the recycling vesicle pool (Fig. 3C,D), consistent with the notion that short-term changes in protein composition at the presynaptic terminal can be mediated by protein degradation alone.

In both invertebrate preparations, proteasome inhibition triggers an increase in synaptic transmission in response to single action potentials, indicating an increase in release probability. In the optical experiments presented here, an increase in release probability would be manifested by an acceleration of dye release, which was not observed (Figs. 1, 2). Thus, although we cannot exclude an (additional) effect on release probability, the activitydependent $60 \%$ increase in the recycling pool at hippocampal synapses appears to be mainly caused by a recruitment of vesicles into the recycling pool.

The prolonged enhancement of the vesicle recycling pool that we observed after proteasome inhibition (Fig. 3B) was somewhat surprising because homeostatic mechanisms of synaptic plasticity might have been expected to counteract the increase in the recycling pool (Turrigiano and Nelson, 2004). Maybe such cor-
C

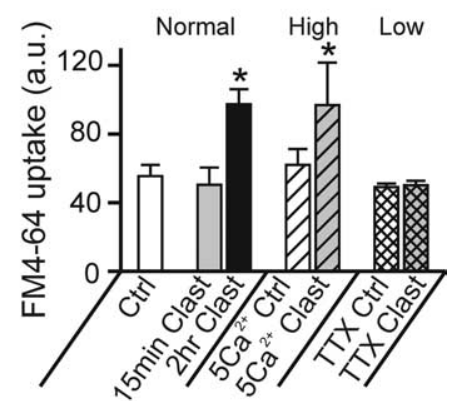

D

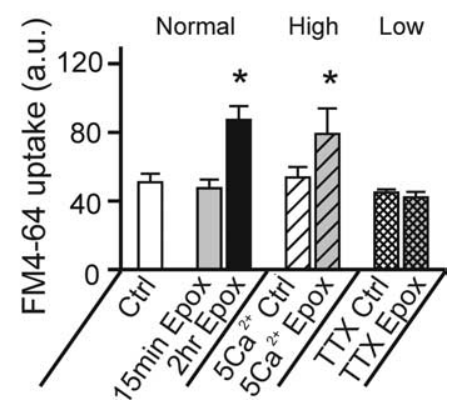

Figure 4. Proteasome inhibition-dependent increase in dye uptake is activity dependent. $\boldsymbol{A}$, Schematic indicating conditions

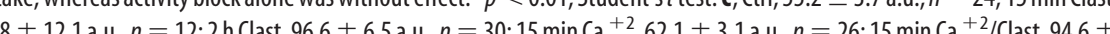

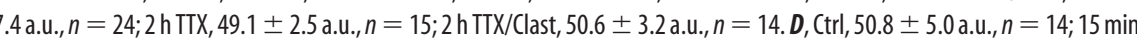

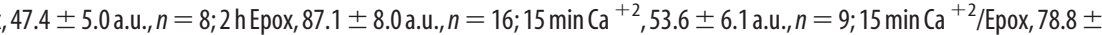
14.9 a.u., $n=9 ; 2 \mathrm{~h} \mathrm{TTX,} 44.9 \pm 1.9$ a.u., $n=7 ; 2 \mathrm{~h} \mathrm{TTX/Epox,} 42.0 \pm 3.2$ a.u., $n=7$. No differences were observed in the percentage of vesicle pool released or kinetics of release (data not shown). Error bars indicate SEM.

rective changes take longer to develop or the increase in the presynaptic vesicle pool might be counterbalanced by postsynaptic changes (Ehlers, 2003; Zhao et al., 2003) to which the dye uptake assay is insensitive. In either case, a prolonged, unbalanced increase in the recycling pool will profoundly affect synaptic function and might ultimately compromise neuronal health.

After 12 DIV, control neurons showed mature levels of vesicle pools (Fig. 3B) (Renger et al., 2001). However, the proteasome inhibitor-dependent increase in the recycling pool became robust only by 16 DIV (Fig. 3A). Obviously, we cannot exclude that this developmental lag (and indeed even the effect of proteasome inhibition) is only observed in vitro. However, the lag might be attributable to a relatively slow maturation of the UPS or of the signal transduction pathways that recruit vesicles. This is consistent with a role for proteasomal activity in fine-tuning established synapses.

The present study indicates that proteasome inhibition increases the pool of recycling vesicles. We define the recycling pool of vesicles as those which actively participate in at least one round of exocytosis and endocytosis in response to a $90 \mathrm{mM} \mathrm{K}^{+}$stimulus (Ryan et al., 1993; Pyle et al., 2000). This pool comprises a morphologically heterogeneous population of vesicles including, but not limited to, vesicles that are docked at the presynaptic membrane (Harata et al., 2001; Rizzoli and Betz, 2004). Our finding that proteasome inhibition enhances the pool of recycling vesi- 
A
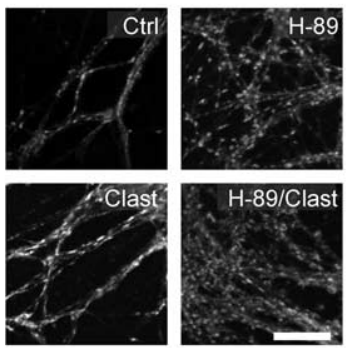

D
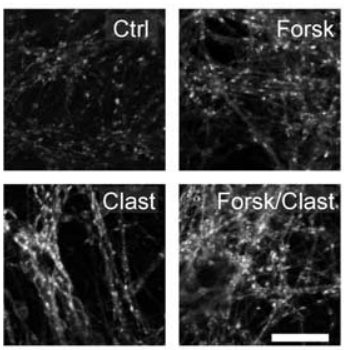

B

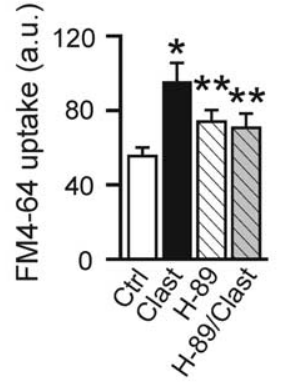

E

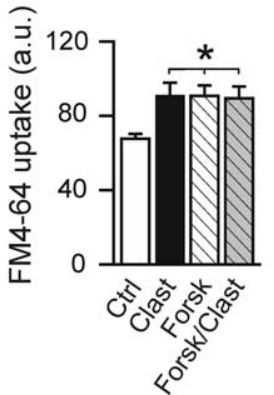

Figure 5. Overlap between the CAMP-PKA pathway and the enhancement of the recycling vesicle pool by clasto-lactacystin $\beta$-lactone. A, Sample images from cultures treated with the PKA inhibitor H-89 (20 $\mu \mathrm{m})$ with and without clasto-lactacystin $\beta$-lactone. After a $1 \mathrm{~h}$ pretreatment with $\mathrm{H}-89$, clasto-lactacystin $\beta$-lactone was added for $2 \mathrm{~h}$, where appropriate. $\mathrm{H}-89$ appears to attenuate the effect of proteasome inhibition. $\boldsymbol{B}$, Quantification of FM4-64 uptake indicates that incubation in $\mathrm{H}-89$ alone is sufficient to elicit a small increase in uptake, although not as pronounced as clasto-lactacystin $\beta$-lactone. However, the effect of the proteasome inhibitor is significantly attenuated in $\mathrm{H}-89$ treated cultures (*relative to Ctrl using two-way ANOVA with Tukey's post hoc analysis at $p<0.05$; ** relative to clasto-lactacystin $\beta$-lactone and ctrl using $t$ test; Ctrl, $55.4 \pm 4.6$ a.u., $n=7$; Clast, $101.8 \pm 9.2$ a.u., $n=7 ;$ H-89, $74.0 \pm 6.2$ a.u., $n=9 ;$ H-89/Clast, $70.6 \pm 7.7$ a.u., $n=10$ ). C, Quantification of FM4-64 uptake after $1 \mathrm{~h}$ pretreatment with the competitive PKA antagonist Rp-CAMPS (100 $\mu \mathrm{m})$ followed by a $2 \mathrm{~h}$ treatment with clastolactacystin $\beta$-lactone, where appropriate. Rp-cAMPS blocks the effect of proteasome inhibition (*relative to Ctrl; two-way ANOVA with Tukey's post hoc analysis at $p<0.05$; Ctrl, $51.1 \pm 5.2$ a.u.; Clast, $76.3 \pm 2.7$ a.u.; Rp-cAMPS, $50.2 \pm 2.4$ a.u.; Rp-CAMPS/ Clast, $55.8 \pm 4.1$ a.u.; $n=9$ in all conditions). $\boldsymbol{D}$, Sample images from cultures treated with the adenylyl-cyclase inhibitor forskolin $(100 \mu \mathrm{m})$ with and without clasto-lactacystin $\beta$-lactone. After a $1 \mathrm{~h}$ pretreatment with forskolin, clasto-lactacystin $\beta$-lactone was added for $2 \mathrm{~h}$, where appropriate. Forskolin alone seems to mimic the effect of clasto-lactacystin $\beta$-lactone. $\boldsymbol{E}$, Quantification of FM4-64 uptake shows that forskolin alone mimics the effect of proteasome inhibition, but the effect is not additive ( ${ }^{*}$ relative to Ctrl; two-way ANOVA with Tukey's post hoc analysis at $p<0.05$; Ctrl, $67.4 \pm 2.7$ a.u., $n=23$; Clast, $90.6 \pm$ 7.1 a.u., $n=18$; Forsk, $90.5 \pm 5.6$ a.u., $n=23$; Forsk/Clast, $89.2 \pm 6.3$ a.u., $n=18$ ). $F$, Quantification of FM4 -64 uptake after $1 \mathrm{~h}$ pretreatment with CAMP agonist Sp-CAMPS $(50 \mu \mathrm{M})$ followed by treatment with clasto-lactacystin $\beta$-lactone, where appropriate. Sp-cAMPS alone mimics the effect of clasto-lactacystin $\beta$-lactone and the effect is not additive (*relative to Ctrl; two-way ANOVA with Tukey's post hoc analysis at $p<0.05$; Ctrl, $50.3 \pm 4.8$ a.u.; Clast, $81.7 \pm 4.1$ a.u.; Sp-cAMPS, $81.1 \pm 2.9$ a.u.; Sp-cAMPS/Clast, $85.6 \pm 8.8$ a.u.; $n=9$ in all conditions).

cles suggests recruitment from a reserve pool. Recruitment of vesicles into the recycling pool appears to be regulated via a cAMP/PKA-dependent pathway (Fig. 5) as has been demonstrated previously at the Drosophila neuromuscular junction (Kuromi and Kidokoro, 2000, 2005). PKA phosphorylation can slow protein degradation through at least two mechanisms: (1) by phosphorylating the protein to be degraded (Gastel et al., 1998; Schomerus et al., 2000), or (2) by phosphorylating components of the UPS (Kotani et al., 1998; Upadhya et al., 2006). It thus seems conceivable that blockade of the proteasome and/or activation of the PKA pathway lead to an increase in otherwise rapidly degraded protein(s) at the presynaptic nerve terminal. For example, proteasome inhibition at the Drosophila NMJ increases levels of the synaptic priming protein Dunc-13, an effect that is abolished by PKA antagonists (Aravamudan and Broadie, 2003). cAMP might additionally act directly through the Rab3interacting molecule (RIM)-Munc 13 priming complex. This complex is activated when cAMP-GEFII (guanine nucleotide

C

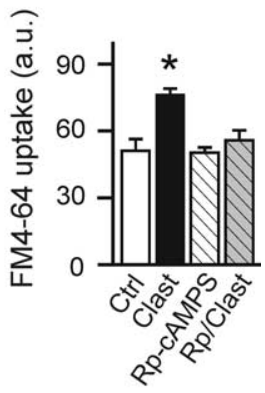

$\mathrm{F}$

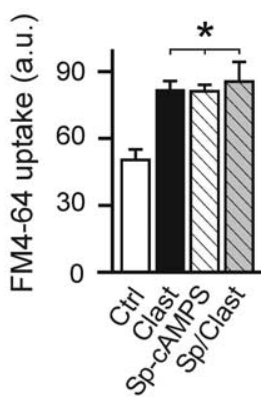

exchange factor II) interacts with RIM (Ozaki et al., 2000), which, in turn, initiates interaction with Munc 13, the mammalian ortholog of Dunc-13 (Betz et al., 2001). Both RIM and Munc 13 have been shown to be important regulators of synaptic vesicle exocytosis (Rosenmund et al., 2002; Calakos et al., 2004).

The proteasome could potentially regulate the expression levels of many proteins implicated in synaptic transmission in addition to Munc 13. Synaptic proteins that have been reported to be ubiquitinated include SNAP-25 (Ma et al., 2005), synaptophysin (Wheeler et al., 2002), syntaxin (Chin et al., 2002), glycosylated $\alpha$-synuclein (Shimura et al., 2001), and synaptotagmin (Huynh et al., 2003). Other candidate proteins that increase synaptic transmission if their intracellular levels increase include the SNARE-complex associated $\alpha$-SNAP protein (DeBello et al., 1995; Xu et al., 1999) and the NCS-1 (neuronal calcium sensor-1), which is quickly degraded, at least under certain conditions (Sippy et al., 2003).

Previous studies revealed a role for the postsynaptic proteasome in activitydependent regulation of synaptic function in hippocampal neurons (Ehlers, 2003; Patrick et al., 2003; Bingol and Schuman, 2006; Karpova et al., 2006). Our findings reveal a novel, activity-dependent presynaptic role of the proteasome. Therefore, we propose that the proteasome may act as a homeostatic regulator of protein levels or function. The activity dependence of the increase in the recycling vesicle pool may be attributable to an activity-dependent increase in presynaptic calcium concentrations that affect the proteasome (Realini and Rechsteiner, 1995) or its targets (Junge et al., 2004). Alternatively, it might be attributable to an increased usage of the recycling machinery during increased activity. We are currently unable to directly distinguish between these two scenarios because manipulations of calcium levels will also affect usage of the recycling machinery. However, our finding that the cAMP-PKA pathway acts in conjunction with proteasome inhibition might point toward an active process rather than enhanced activity simply allowing for proteasomal action at each round of recycling.

Synthetic and naturally occurring proteasome inhibitors are abundantly present in the environment (Kisselev and Goldberg, 2001). The acute effects of proteasome inhibition on synaptic transmission reported here may, thus, be relevant to acute exposure to environmental toxins and/or might represent early stages of chronic exposure (McNaught et al., 2004; Sun et al., 2005; Betarbet et al., 2006). An increase in the recycling pool of vesicles will fundamentally change neuronal signaling and possibly contribute to cellular dysfunction.

In conclusion, our studies point to the importance of the proteasome as a negative feedback regulator of synaptic transmis- 
sion. Acute modulation of proteasome activity under physiological conditions could occur through at least three different mechanisms: (1) regulation of proteasomal subunit expression (James et al., 2006), (2) regulation of proteasomal activity through $\mathrm{Ca}^{2+}$ (Realini and Rechsteiner, 1995) or through phosphorylation (Kotani et al., 1998; Upadhya et al., 2006), and (3) via phosphorylation of proteasomal target proteins (Gastel et al., 1998; Schomerus et al., 2000). The proteasome, through its dynamic role as a protein modulator, may thus point toward a novel mechanism of homeostatic regulation at the nerve terminal.

\section{References}

Aravamudan B, Broadie K (2003) Synaptic Drosophila UNC-13 is regulated by antagonistic G-protein pathways via a proteasome-dependent degradation mechanism. J Neurobiol 54:417-438.

Beaumont V, Zhong N, Fletcher R, Froemke RC, Zucker RS (2001) Phosphorylation and local presynaptic protein synthesis in calcium- and calcineurin-dependent induction of crayfish long-term facilitation. Neuron 32:489-501.

Betarbet R, Canet-Aviles RM, Sherer TB, Mastroberardino PG, McLendon C, Kim J-H, Lund S, Na H-M, Taylor G, Bence NF, Kopito R, Seo BB, Yagi T, Yagi A, Klinefelter G, Cookson MR, Greenamyre JT (2006) Intersecting pathways to neurodegeneration in Parkinson's disease: effects of the pesticide rotenone on DJ-1, $\alpha$-synuclein, and the ubiquitin-proteasome system. Neurobiol Dis 22:404-420.

Betz A, Thakur P, Junge HJ, Ashery U, Rhee JS, Scheuss V, Rosenmund C, Rettig J, Brose N (2001) Functional interaction of the active zone proteins Munc13-1 and RIM1 in synaptic vesicle priming. Neuron 30:183-196.

Betz WJ, Bewick GS (1992) Optical analysis of synaptic vesicle recycling at the frog neuromuscular junction. Science 255:200-203.

Bingol B, Schuman EM (2005) Synaptic protein degradation by the ubiquitin proteasome system. Curr Opin Neurobiol 15:536-541.

Bingol B, Schuman EM (2006) Activity-dependent dynamics and sequestration of proteasomes in dendritic spines. Nature 441:1144-1148.

Burbea M, Dreier L, Dittman JS, Grunwald ME, Kaplan JM (2002) Ubiquitin and AP180 regulate the abundance of GLR-1 glutamate receptors at postsynaptic elements in C. elegans. Neuron 35:107-120.

Calakos N, Schoch S, Sudhof TC, Malenka RC (2004) Multiple roles for the active zone protein RIMlalpha in late stages of neurotransmitter release. Neuron 42:889-896.

Chen H, Polo S, Di Fiore PP, De Camilli PV (2003) Rapid $\mathrm{Ca}^{2+}$-dependent decrease of protein ubiquitination at synapses. Proc Natl Acad Sci USA 100:14908-14913.

Chin LS, Vavalle JP, Li L (2002) Staring, a novel E3 ubiquitin-protein ligase that targets syntaxin 1 for degradation. J Biol Chem 277:35071-35079.

Cline H (2003) Synaptic plasticity: importance of proteasome-mediated protein turnover. Curr Biol 13:R514-R516.

Colledge M, Snyder EM, Crozier RA, Soderling JA, Jin Y, Langeberg LK, Lu H, Bear MF, Scott JD (2003) Ubiquitination regulates PSD-95 degradation and AMPA receptor surface expression. Neuron 40:595-607.

DeBello WM, V OC, Dresbach T, Whiteheart SW, Wang SS, Schweizer FE, Betz H, Rothman JE, Augustine GJ (1995) SNAP-mediated proteinprotein interactions essential for neurotransmitter release. Nature 373:626-630.

Efron B, Tibshirani R (1991) Statistical data analysis in the computer age. Science 253:390-395.

Ehlers MD (2003) Activity level controls postsynaptic composition and signaling via the ubiquitin-proteasome system. Nat Neurosci 6:231-242.

Fenteany G, Standaert RF, Reichard GA, Corey EJ, Schreiber SL (1994) A beta-lactone related to lactacystin induces neurite outgrowth in a neuroblastoma cell line and inhibits cell cycle progression in an osteosarcoma cell line. Proc Natl Acad Sci USA 91:3358-3362.

Gastel JA, Roseboom PH, Rinaldi PA, Weller JL, Klein DC (1998) Melatonin production: proteasomal proteolysis in serotonin $\mathrm{N}$-acetyltransferase regulation. Science 279:1358-1360.

Giuditta A, Kaplan BB, van Minnen J, Alvarez J, Koenig E (2002) Axonal and presynaptic protein synthesis: new insights into the biology of the neuron. Trends Neurosci 25:400-404.

Glickman MH, Ciechanover A (2002) The ubiquitin-proteasome proteolytic pathway: destruction for the sake of construction. Physiol Rev 82:373-428.

Harata N, Ryan TA, Smith SJ, Buchanan J, Tsien RW (2001) Visualizing recycling synaptic vesicles in hippocampal neurons by FM 1-43 photoconversion. Proc Natl Acad Sci USA 98:12748-12753.

Hegde AN (2004) Ubiquitin-proteasome-mediated local protein degradation and synaptic plasticity. Prog Neurobiol 73:311-357.

Huynh DP, Scoles DR, Nguyen D, Pulst SM (2003) The autosomal recessive juvenile Parkinson disease gene product, parkin, interacts with and ubiquitinates synaptotagmin XI. Hum Mol Genet 12:2587-2597.

James AB, Conway AM, Morris BJ (2006) Regulation of the neuronal proteasome by Zif268 (Egr1). J Neurosci 26:1624-1634.

Junge HJ, Rhee JS, Jahn O, Varoqueaux F, Spiess J, Waxham MN, Rosenmund C, Brose N (2004) Calmodulin and Munc13 form a Ca ${ }^{2+}$ sensor/effector complex that controls short-term synaptic plasticity. Cell 118:389-401.

Juo P, Kaplan JM (2004) The anaphase-promoting complex regulates the abundance of GLR-1 glutamate receptors in the ventral nerve cord of $C$. elegans. Curr Biol 14:2057-2062.

Karpova A, Mikhaylova M, Thomas U, Knopfel T, Behnisch T (2006) Involvement of protein synthesis and degradation in long-term potentiation of Schaffer collateral CA1 synapses. J Neurosci 26:4949-4955.

Kidokoro Y, Kuromi H, Delgado R, Maureira C, Oliva C, Labarca P (2004) Synaptic vesicle pools and plasticity of synaptic transmission at the Drosophila synapse. Brain Res Brain Res Rev 47:18-32.

Kisselev AF, Goldberg AL (2001) Proteasome inhibitors: from research tools to drug candidates. Chem Biol 8:739-758.

Kotani S, Tugendreich S, Fujii M, Jorgensen PM, Watanabe N, Hoog C, Hieter P, Todokoro K (1998) PKA and MPF-activated polo-like kinase regulate anaphase-promoting complex activity and mitosis progression. Mol Cell 1:371-380.

Kuromi H, Kidokoro Y (1999) The optically determined size of exo/endo cycling vesicle pool correlates with the quantal content at the neuromuscular junction of Drosophila larvae. J Neurosci 19:1557-1565.

Kuromi H, Kidokoro Y (2000) Tetanic stimulation recruits vesicles from reserve pool via a cAMP-mediated process in Drosophila synapses. Neuron 27:133-143.

Kuromi H, Kidokoro Y (2003) Two synaptic vesicle pools, vesicle recruitment and replenishment of pools at the Drosophila neuromuscular junction. J Neurocytol 32:551-565.

Kuromi H, Kidokoro Y (2005) Exocytosis and endocytosis of synaptic vesicles and functional roles of vesicle pools: lessons from the Drosophila neuromuscular junction. Neuroscientist 11:138-147.

Ma Z, Portwood N, Foss A, Grill V, Bjorklund A (2005) Evidence that insulin secretion influences SNAP-25 through proteasomal activation. Biochem Biophys Res Commun 329:1118-1126.

McNaught KS, Perl DP, Brownell AL, Olanow CW (2004) Systemic exposure to proteasome inhibitors causes a progressive model of Parkinson's disease. Ann Neurol 56:149-162.

Murphey RK, Godenschwege TA (2002) New roles for ubiquitin in the assembly and function of neuronal circuits. Neuron 36:5-8.

Ozaki N, Shibasaki T, Kashima Y, Miki T, Takahashi K, Ueno H, Sunaga Y, Yano H, Matsuura Y, Iwanaga T, Takai Y, Seino S (2000) cAMP-GEFII is a direct target of cAMP in regulated exocytosis. Nat Cell Biol 2:805-811.

Patrick GN (2006) Synapse formation and plasticity: recent insights from the perspective of the ubiquitin proteasome system. Curr Opin Neurobiol 16:90-94.

Patrick GN, Bingol B, Weld HA, Schuman EM (2003) Ubiquitin-mediated proteasome activity is required for agonist-induced endocytosis of GluRs. Curr Biol 13:2073-2081.

Poskanzer KE, Marek KW, Sweeney ST, Davis GW (2003) Synaptotagmin I is necessary for compensatory synaptic vesicle endocytosis in vivo. Nature 426:559-563.

Pyle JL, Kavalali ET, Piedras-Renteria ES, Tsien RW (2000) Rapid reuse of readily releasable pool vesicles at hippocampal synapses. Neuron 28:221-231.

Realini C, Rechsteiner M (1995) A proteasome activator subunit binds calcium. J Biol Chem 270:29664-29667.

Renger JJ, Egles C, Liu G (2001) A developmental switch in neurotransmitter flux enhances synaptic efficacy by affecting AMPA receptor activation. Neuron 29:469-484. 
Rizzoli SO, Betz WJ (2004) The structural organization of the readily releasable pool of synaptic vesicles. Science 303:2037-2039.

Rosenmund C, Sigler A, Augustin I, Reim K, Brose N, Rhee JS (2002) Differential control of vesicle priming and short-term plasticity by Munc13 isoforms. Neuron 33:411-424.

Ryan TA, Reuter H, Wendland B, Schweizer FE, Tsien RW, Smith SJ (1993) The kinetics of synaptic vesicle recycling measured at single presynaptic boutons. Neuron 11:713-724.

Schikorski T, Stevens CF (2001) Morphological correlates of functionally defined synaptic vesicle populations. Nat Neurosci 4:391-395.

Schomerus C, Korf HW, Laedtke E, Weller JL, Klein DC (2000) Selective adrenergic/cyclic AMP-dependent switch-off of proteasomal proteolysis alone switches on neural signal transduction: an example from the pineal gland. J Neurochem 75:2123-2132.

Shimura H, Schlossmacher MG, Hattori N, Frosch MP, Trockenbacher A, Schneider R, Mizuno Y, Kosik KS, Selkoe DJ (2001) Ubiquitination of a new form of alpha-synuclein by parkin from human brain: implications for Parkinson's disease. Science 293:263-269.

Sippy T, Cruz-Martin A, Jeromin A, Schweizer FE (2003) Acute changes in short-term plasticity at synapses with elevated levels of neuronal calcium sensor-1. Nat Neurosci 6:1031-1038.

Speese SD, Trotta N, Rodesch CK, Aravamudan B, Broadie K (2003) The ubiquitin proteasome system acutely regulates presynaptic protein turnover and synaptic efficacy. Curr Biol 13:899-910.
Sun F, Anantharam V, Latchoumycandane C, Kanthasamy A, Kanthasamy AG (2005) Dieldrin induces ubiquitin-proteasome dysfunction in alphasynuclein overexpressing dopaminergic neuronal cells and enhances susceptibility to apoptotic cell death. J Pharmacol Exp Ther 315:69-79.

Turrigiano GG, Nelson SB (2004) Homeostatic plasticity in the developing nervous system. Nat Rev Neurosci 5:97-107.

Upadhya SC, Ding L, Smith TK, Hegde AN (2006) Differential regulation of proteasome activity in the nucleus and the synaptic terminals. Neurochem Int 48:296-305.

Wheeler TC, Chin LS, Li Y, Roudabush FL, Li L (2002) Regulation of synaptophysin degradation by mammalian homologues of seven in absentia. J Biol Chem 277:10273-10282.

Wu KY, Hengst U, Cox LJ, Macosko EZ, Jeromin A, Urquhart ER, Jaffrey SR (2005) Local translation of RhoA regulates growth cone collapse. Nature 436:1020-1024.

Xu T, Ashery U, Burgoyne RD, Neher E (1999) Early requirement for alphaSNAP and NSF in the secretory cascade in chromaffin cells. EMBO J 18:3293-3304.

Yi JJ, Ehlers MD (2005) Ubiquitin and protein turnover in synapse function. Neuron 47:629-632.

Zhao Y, Hegde AN, Martin KC (2003) The ubiquitin proteasome system functions as an inhibitory constraint on synaptic strengthening. Curr Biol 13:887-898. 